Pacific Journal of Mathematics

CLASSES WITHOUT THE AMALGAMATION PROPERTY 


\section{CLASSES WITHOUT THE AMALGAMATION PROPERTY}

\section{S. D. COMER}

The contents of this paper belong to the general algebraic theory of those algebras which are studied in connection with algebraic logic. The main results, Theorems 1 and 2 , give sufficient conditions for the amalgamation and the embedding property to fail in a class of Boolean algebras with operators. As a corollary, for $1<\alpha<\omega$, the amalgamation property fails in the class of all (representable) cylindric algebras of dimension $\alpha$ and in the class of all (representable) polyadic (equality) algebras of dimension $\alpha$. Thus, there are finitely axiomatizable equational classes of Boolean algebras with operators for which the amalgamation property fails.

The amalgamation and embedding properties have proved to be an extremely useful tool in model-theoretic investigations (references may be found in Jónsson [8]) and in the development of algebraic analogues to logical theorems (cf., Daigneault [2]). Recently, Don Pigozzi has shown that for certain classes of cylindric algebras the amalgamation property is equivalent to a certain algebraic form of Craig's Interpolation Theorem.

The first answer to Jónsson's question in [8] concerning whether or not there exist an equational class of Boolean algebras with operators for which the amalgamation property fails was given in R. McKenzie [12]. Using Lyndon algebras McKenzie showed that this property fails for the class $R R A$ of all representable relation algebras and that the embedding property fails in a nontrivial way for the classes $R R A$ and $R A$ (the class of all relation algebras). We also obtain these results. The negative results obtained in Corollary 4 complement the work of A. Daigneault and J. Johnson. In [2] Daigneault shows that the amalgamation property holds for the class of all locally finite polyadic (equality) algebras of infinite dimension. Johnson [7] has extended Daigneault's work to show that the amalgamation property holds for the class of all polyadic and polyadic equality algebras of infinite dimension. Whether or not it holds for the class $C A_{\alpha}, \alpha \geqq \omega$, in general, is not known.

It is shown in Eos [10] that a necessary condition for any two algebras of a class to have a common extension in the class is that all algebras of the class have isomorphic minimal subalgebras. An example, due to Białynicki-Birula, given in Łos [11] shows the condition is not sufficient even for equational classes. The classes listed in Corollary 5 provide further examples of this phenomenon. In $\S 3$ we 
give a necessary and sufficient condition for two algebras of a class to have a common extension in the class. This condition is then used to show we cannot remove one of our restrictions on classes of algebras from the hypothesis of Theorem 2.

1. Throughout this paper we assume familiarity with the basic notions and results of mathematical logic, set theory and general algebra. In this section we will briefly introduce and discuss some of our terminology.

We assume that the ordinal numbers have been defined in such a way that each ordinal is the set of all smaller ordinals. The cardinality of a set $X$ is denoted by $|X|$. The set of all functions from a set $X$ into a set $Y$ is denoted by ${ }^{x} Y$; for a binary relation $R$ on a set $X$ and $x$ in the domain of $R$, we denote $\{y \in X:(x, y) \in R\}$ by $R^{*} x$. If $f$ is a function and $X$ is a subset of the domain of $f$, we denote by $f \mid X$ the function with domain $X$ which is equal to $f$ on $X$. We use the usual arrow notation $\mathfrak{A} \rightarrow \mathfrak{B}, \mathfrak{A} \succ \mathfrak{B}$, etc., to denote respectively, a homomorphism from an algebra $\mathfrak{A}$ into an algebra $\mathfrak{B}$, a monomorphism from $\mathfrak{U}$ into $\mathfrak{B}$, etc. For a homomorphism $f$ on an algebra $\mathfrak{U}$ we denote the homomorphic image of $\mathfrak{A}$ by $f^{*} \mathfrak{A}$.

Let $K$ be a class of similar algebras. We say that $K$ has the amalgamation property if for ever $\mathfrak{U}, \mathfrak{B}_{0}, \mathfrak{B}_{1}, \in K$ and all monomorphisms $f_{i}: \mathfrak{U} \succ \mathfrak{B}_{i}(i=0,1)$, there exist a algebra $\mathbb{E} \in K$ and monomorphisms $g_{i}: \mathfrak{B}_{i} \nrightarrow \mathfrak{B}$ such that the diagram

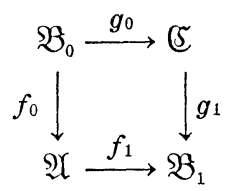

commutes, i.e., $g_{0} f_{0}=g_{1} f_{1}$.

We say that $K$ has the embedding or common extension property if for every $\mathfrak{A}_{0}, \mathfrak{A}_{1} \in K$ there exist a $\mathfrak{B} \in K$ and monomorphisms $f_{0}: A_{0} \nrightarrow \mathfrak{B}$ and $f_{1}: \mathfrak{A}_{1} \nrightarrow \mathfrak{B}$.

For an algebra $\mathfrak{A}$ we denote by $\stackrel{n-\mathfrak{A}}{\tau}$ the $n$-ary operation on $\mathfrak{A}$ induced by the term $\tau$ in the language of the similarity type of $\mathfrak{A}$ and with variables included among $v_{0}, \cdots, v_{n-1}$. Now suppose $K$ and $L$ are classes of algebras of similarity type $\mu$ and $\mu^{\prime}$ respectively. Suppose $\tau$ is a function from dom $\mu^{\prime}$ into the set of terms of the language of $\mu$ with the property that $\tau_{i}$ has variables included among $v_{0}, \cdots, v_{\mu^{\prime}(i)-1}$ for all $i \in \operatorname{dom} \mu^{\prime}$. We say that $\tau$ is an equational definitional embedding (e.d.e.) of $K$ into $L$ if the algebra

$$
\tau^{*} \mathfrak{A}=\left\langle A, \stackrel{\mu^{\prime \prime}(i)-\mathfrak{Y}}{\tau_{i}}\right\rangle_{i \in \mathrm{dom} \mu^{\prime}}
$$


belongs to $L$ for all algebras $\mathfrak{U}$ in $K$. It should be observed that if $K$ and $L$ are considered as a categories in the natural way then the correspondence of $\mathfrak{U} \in K$ to $\tau^{*} \mathfrak{U} \in L$ associated with an e.d.e. $\tau$ is the object map of functor from $K$ into $L$.

We now give a few basic definitions from the theories of cylindrification and cylindric algebras. For additional information see Henkin and Tarski [6].

A cylindric algebra of dimensional $\alpha\left(a C A_{\alpha}\right)$ is a system

$$
\mathfrak{U}=\left\langle A,+, \cdot,-, 0,1, c_{\kappa}, d_{\kappa \lambda}\right\rangle_{\kappa, \lambda<\alpha}
$$

where $\alpha$ is an ordinal, $0,1, d_{\kappa \lambda} \in A,-, c_{\kappa} \in{ }^{A} A,+, \cdot \in{ }^{A \times A} A$ and the following conditions hold for all $x, y \in A, \kappa, \lambda, \mu<\alpha$ :

$\left(C_{0}\right)$ the system $\langle A,+, \cdot,-, 0,1\rangle$ is a Boolean algebra;

$\left(C_{1}\right) \quad C_{\kappa} 0=0$;

$\left(C_{2}\right) \quad x \leqq c_{\kappa} x$

$\left(C_{3}\right) \quad c_{\kappa}\left(x \cdot c_{\kappa} y\right)=c_{\kappa} x \cdot c_{\kappa} y$;

$\left(C_{4}\right) \quad c_{\kappa} c_{\lambda} x=c_{k} c_{n} x$

$\left(C_{5}\right) \quad d_{\kappa \kappa}=1$;

$\left(C_{6}\right)$ if $k \neq \lambda, \mu$, then $d_{\lambda^{\mu}}=c_{\kappa}\left(d_{\lambda \kappa} \cdot d_{\kappa^{\mu}}\right)$;

$\left(C_{7}\right)$ if $\kappa \neq \lambda$, then $c_{\kappa}\left(d_{\kappa \lambda} \cdot x\right) \cdot c_{\kappa}\left(d_{\kappa \lambda} \cdot-x\right)=0$.

For a $C A_{\alpha} \mathfrak{X}$ and a finite subset $\Gamma$ of $\alpha$, say $\Gamma=\left\{\lambda_{0}, \cdots, \lambda_{n-1}\right\}$ we let $c_{(\Gamma)} x=c_{\lambda_{0}} c_{\lambda_{1}} \cdots c_{\lambda_{n-1}} x$ for all $x \in A$. We call $\prod_{\kappa, \lambda<\alpha} d_{\kappa \lambda}$ the main diagonal of $\mathfrak{X}$ when it exists and occasionary denote it by $d^{\mathscr{U}}$ or just $d$. We shall discuss neither the elementary arithmetic for cylindric algebras nor the familiar algebraic concepts of subalgebras, homomorphisms, subdirect and direct products of cylindric algebras, nor the notion of a simple $C A_{\alpha}$. For information on these concepts see Henkin and Tarski [6].

For a set $U \neq 0$ and an ordinal $\alpha$, consider the system

$$
\left\langle\mathrm{S}\left({ }^{\alpha} U\right), \cup, \cap, \sim, 0,{ }^{\alpha} U, C_{\kappa}, D_{\kappa \lambda}\right\rangle_{\kappa, \lambda<\alpha}
$$

where $S\left({ }^{\alpha} U\right)$ is the set of all subsets of ${ }^{\alpha} U, U, \cap$, and $\sim$ are the usual set-theoretic operations, and for $\kappa, \lambda<\alpha$ and $X \cong{ }^{\alpha} U, D_{k \lambda}$ and $C_{\kappa} X$ are defined by

$$
\begin{aligned}
D_{\kappa \lambda} & =\left\{y \in{ }^{\alpha} U: y_{\kappa}=y_{\lambda}\right\}, \text { and } \\
C_{\kappa} X & =\left\{y \in{ }^{\alpha} U: y|(\alpha \sim\{\kappa\})=z|(\alpha \sim\{\kappa\}) \text { for some } z \in X\right\} .
\end{aligned}
$$

The above system is called the full cylindric set algebra of dimension $\alpha$ and base $U$ and is denoted by $\mathfrak{T}(\alpha, U)$. A $C A_{\alpha} \mathfrak{A}$ is representable (an $\left.R C A_{\alpha}\right)$ if $\mathfrak{A}$ is isomorphic to a subdirect product of subalgebras of algebras $\mathfrak{T}(\alpha, U)$.

An algebra $\mathfrak{A}=\left\langle A,+, \cdot,-, 0,1, c_{\kappa}\right\rangle_{\kappa<\alpha}$ which satisfies $\left(C_{0}\right)-\left(C_{4}\right)$ is called a cylindrification algebra of dimension $\alpha\left(a C y_{\alpha}\right)$. The notation 
and definitions given above extend in the obvious way to cylindrification algebras. In particular we denote the full cylindrification set algebra of dimension $\alpha$ and base $U$ by $\mathfrak{A}_{c}(\alpha, U)$ and the class of all representable $C y_{\alpha}$ 's by $R C y_{\alpha}$.

If a $C A_{\alpha} \mathfrak{U}$ has a simple minimal subalgebra, we say that $\mathfrak{U}$ has characteristic 0 if

$$
0 \neq c_{(\lambda)} \Pi_{i, j \in \lambda, i \neq j}-d_{i j}
$$

for all $\lambda<(\alpha+1) \cap \omega$. We may alternatively describe this class of algebras as the class of $C A_{\alpha}$ 's whose minimal subalgebra is isomorphic to the minimal subalgebra of $\mathfrak{N}(\alpha, \alpha)$. We can define the class of polyadic equality algebras of characteristic 0 in a similar manner.

We now introduce conditions (A) and (B) on classes $K$ of Boolean algebras with operators. Suppose $\tau$ is an e.d.e. of $K$ into the class $C y_{\alpha}$.

Condition (A). There exist sets $U_{j} \neq 0$, algebras $\mathfrak{A}\left(U_{j}\right), \mathfrak{M} \in K$ and monomorphisms $g: \mathfrak{M} \nrightarrow \mathfrak{A}\left(U_{j}\right)$ for $j=0,1$ such that

(i ) $\alpha \leqq\left|U_{0}\right|<\left|U_{1}\right|$ and $\left|U_{0}\right|<\omega$;

(ii) for $j=0,1, \tau^{*} \mathfrak{A}\left(U_{j}\right)=\mathfrak{A}_{c}\left(\alpha, U_{j}\right)$, the full set $C y_{\alpha}$ with base $U_{j}$;

(iii) $D_{j} \in A\left(U_{j}\right)$ for $j=0,1$, and $g_{1} \circ g_{0}^{-1}$ is an isomorphism from $g_{0}^{*} \mathfrak{M}$ onto $g_{1}^{*} \mathfrak{M}$ such that $g_{1} g_{0}^{-1} D_{0}=D_{1}$, where $D_{j}$ is the main diagonal of $\mathfrak{U}\left(\alpha, U_{j}\right)$ for $j=0,1$.

Now suppose that $\tau$ is an e.d.e. of $K$ into the class $C A_{\alpha}$.

Condition (B). There exist sets $U_{j} \neq 0$ and algebras $\mathfrak{A}\left(U_{j}\right) \in K$ for $j=0,1$ such that

(i ) $\alpha \leqq\left|U_{0}\right|<\left|U_{1}\right|$ and $\left|U_{0}\right|<\omega$;

(ii) for $j=0,1 \tau^{*} \mathfrak{A}\left(U_{j}\right)=\mathfrak{A}\left(\alpha, U_{j}\right)$, the full set $C A_{\alpha}$ with base $U_{j}$.

2. We now state the main results of this paper.

THEOREM 1. The amalgamation property fails for any class $K$ which satisfies Condition $(A)$ for some e.d.e. $\tau$ of $K$ into the class $C y_{\alpha}$ for some $\alpha$ where $1<\alpha<\omega$.

THEOREM 2. The embedding property fails for any class $K$ which satisfies condition (B) for some e.d.e. $\tau$ of $K$ into the class $C A_{\alpha}$ for some $\alpha$ where $1<\alpha<\omega$.

The proofs depend on the following lemma.

LemmA 3. Suppose $1<\alpha \leqq\left|U_{0}\right|<\left|U_{1}\right|$ and $\left|U_{0}\right|<\omega$. Then there does not exist a $C y_{\alpha} \mathfrak{B}$ and isomorphisms $f_{0}, f_{1}$ such that for $i=0,1 f_{i}$ embeds the full $C y_{\alpha} \mathfrak{A}\left(\alpha, U_{i}\right)$ into $\mathfrak{B}$ and $f_{0}\left(D_{0}\right)=f_{1}\left(D_{1}\right)$ where 
$D_{i}$ is the main diagonal of $\mathfrak{A}\left(\alpha, U_{i}\right)$.

Proof. Suppose there exist such isomorphisms $f_{i}(i=0,1)$ and a $C y_{\alpha} \mathfrak{B}$. Let $f_{0}\left(D_{0}\right)=d=f_{1}\left(D_{1}\right)$. Since $\mathfrak{A}_{c}\left(\alpha, U_{0}\right)$ and $\mathfrak{A}_{c}\left(\alpha, U_{1}\right)$ are simple we may assume that $\mathfrak{B}$ is also simple.

It is easy to verify the following facts about $\mathfrak{I}_{c}(\alpha, X)$ where $|X|=\kappa$.

(1) There exist $\kappa$ atoms, say $a_{i}$ for $i<\kappa$, in $\mathfrak{A}_{c}(\alpha, X)$ such that

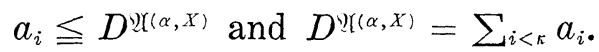

(1') All atoms of $\mathfrak{Y}_{c}(\alpha, X)$ are of the form $\prod_{i<\alpha} c_{(\alpha \sim\{i\}} a_{\phi(i)}$ for $\phi \in{ }^{\alpha} \kappa ; a_{j}=\prod_{i<\alpha} c_{(\alpha \sim i i)} a_{j}$ for $j<\kappa$; and if $j_{0}, j_{1}<\alpha, \phi \in{ }^{\alpha} \kappa$ with $\phi\left(j_{0}\right) \neq$ $\phi\left(j_{1}\right)$, then

$$
\prod_{i<\alpha} c_{(\alpha \sim\{i\})} a_{\phi(i)} \leqq-D^{\mathscr{Y}(\alpha, X)} .
$$

Letting $X$ be $U_{0}$ or $U_{1}$ and applying the isomorphisms $f_{0}, f_{1}$ we obtain from (1) and $\left(1^{\prime}\right)$ the following:

(2) There exist $\kappa=\left|U_{0}\right|$ atoms, say $a_{i}$ for $i<\kappa$, in $f_{0}^{*}\left(\mathfrak{A}_{c}\left(\alpha, U_{0}\right)\right)$ such that $a_{i} \leqq d$ and $\sum_{i<\kappa} a_{i}=d$ (since $\left.\kappa<\omega\right)$.

$\left(2^{\prime}\right)$ same as $\left(1^{\prime}\right)$ with $\mathfrak{P}_{c}(\alpha, X)$ replaced by $f_{0}^{*}\left(\mathfrak{H}\left(\alpha, U_{0}\right)\right)$ and $D^{2(\alpha, X)}$ replaced by $d$.

(3) There exist $\lambda=\left|U_{1}\right|$ atoms, say $b_{i}$ for $i<\lambda$, in $f_{1}^{*}\left(\mathfrak{U}_{c}\left(\alpha, U_{1}\right)\right)$ such that $b_{i} \leqq d$.

$\left(3^{\prime}\right)$ same as $\left(1^{\prime}\right)$ with $\mathfrak{A}_{c}(\alpha, X)$ replaced by $f_{1}^{*}\left(\mathfrak{A}_{c}\left(\alpha, U_{1}\right)\right)$ and $D^{\mathfrak{A}(\alpha, X)}$ replaced by $d$.

By hypothesis $\kappa<\lambda$, so from (2) and (3) we may choose $j<$ $\kappa, m, n<\lambda, m \neq n$ such that

(4) $a_{j} \cdot b_{m} \neq 0$ and $a_{i} \cdot b_{n} \neq 0$.

We now show

( 5 ) $a_{j} \cdot-d \neq 0$.

Let $x=c_{(\alpha \sim\{m\})}\left(a_{j} \cdot b_{m}\right) \cdot \prod_{i<\alpha, i \neq m} c_{\langle\alpha \sim\{i\}}\left(a_{j} \cdot b_{n}\right)$.

From $\left(2^{\prime}\right)$ and $\left(3^{\prime}\right)$ it is clear that $x \leqq a_{j} \cdot-d$. Suppose $x=0$.

Then

$$
\begin{aligned}
0 & =c_{\{\alpha \sim\{m\})} x \\
& =c_{(\alpha \sim\{m\})}\left(a_{j} \cdot b_{m}\right) \cdot c_{\langle\alpha \sim\{m\})}\left[\prod_{i<\alpha, i \neq m} c_{(\alpha \sim i i)}\left(a_{j} \cdot b_{n}\right)\right] \\
& =c_{(\alpha \sim\{m\})}\left(a_{j} \cdot b_{m}\right) \cdot \prod_{i<\alpha, i \neq m} c_{(\alpha)}\left(a_{j} \cdot b_{n}\right) .
\end{aligned}
$$

Since $\mathfrak{B}$ is simple and $a_{j} \cdot b_{n} \neq 0$ by $(4), c_{(\alpha)}\left(a_{j} \cdot b_{n}\right)=1$. Hence

$$
c_{(\alpha \sim\{m\})}\left(a_{j} \cdot b_{m}\right)=0
$$

contradicting (4). Thus $x \neq 0$ and (5) holds. However, (5) contradicts $a_{j} \leqq d$ of (2); hence Lemma 3 is proven.

Proof of Theorem 1. Suppose $K$ is as in the statement of the 
theorem and the amalgamation property holds in $K$. Then there exist $\mathfrak{B} \in K$, isomorphisms $f_{0}$ and $f_{1}$ such that the diagram

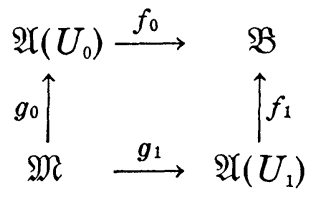

commutes. By properties of terms, the diagram

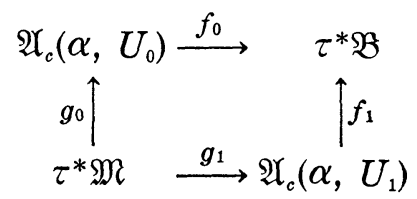

commutes. By (A) (iii), $f_{0}\left(D_{0}\right)=f_{1}\left(D_{1}\right)$. This situation is impossible by Lemma 3 .

Proof of Theorem 2. Let $K$ be as in the statement of the theorem. Suppose there is a $\mathfrak{B} \in K$ and monomorphisms $f_{0}$ and $f_{1}$ such that $f_{i}: \mathfrak{A}\left(U_{i}\right) \nrightarrow \mathfrak{B}$ for $i=0,1$. Then $f_{i}: \mathfrak{A}\left(\alpha, U_{i}\right) \nrightarrow \tau^{*} \mathfrak{B}$ for $i=0,1$. Suppose $\tau_{\kappa \lambda}$ is the term in the language of $K$ which defines $d_{\kappa \lambda}$. Then for the cylindric monomorphisms $f_{i}, f_{i}\left(D_{\kappa \lambda}\right)={ }^{0} \bar{\tau}_{\kappa \lambda}^{\mathfrak{B}}$ for all $i=0,1$ and $\kappa, \lambda<\alpha$. Thus $f_{0}\left(D_{0}\right)=\prod_{\kappa, \lambda<\alpha}{ }^{0} \bar{\tau}_{\kappa \lambda}^{\Re}=f_{1}\left(D_{1}\right)$ where $D_{i}$ is the main diagonal of $\mathfrak{A}\left(\alpha, U_{i}\right)$. Such an embedding of the cylindrification part is impossible by Lemma 3; thus the theorem holds.

Except for the claim dealing with the class of projective algebras the following corollaries are immediate. That Theorems 1 and 2 apply to the classes $(R R A) R A$ of (representable) relation algebras follows from the relationship between $R A^{\prime}$ s and $C A_{2}^{\prime}$ s found on p. 135 of Jónsson and Tarski [9]. For the class of an $R A$, see Definition 4.34 of Jónsson and Tarski [9]; for information concerning polyadic and polyadic equality algebras see Halmos [5] and for information on projective algebras see Everett and Ulam [4].

COROLLARY 4. The amalgamation property fails for the following classes of algebras where $1<\alpha<\omega: C A_{\alpha}, R C A_{\alpha}, C y_{\alpha}, R C y_{\alpha}$, representable polyadic and polyadic equality algebras of dimension $\alpha$, polyadic and polyadic equality algebras of dimension $\alpha, R A, R R A$, and projective algebras. ${ }^{1}$

The following proof that Theorem 1 applies to the class $K$ of

1 This result concerning projective algebras was pointed out by the referee. 
projective algebras is due to the referee. Define $\tau_{0}$ as $P_{0} 1 \square P_{1} v_{0}, \tau_{1}$ as $P_{0} v_{0} \square P_{1} 1$, and $\tau$ natural for the Boolean operations. Then $\tau$ is an e.d.e. of $K$ into $C y_{2}$. Suppose $U_{0} \subset U_{1},\left|U_{0}\right|=3, a \in U_{0}$ and let $\mathfrak{A}\left(U_{j}\right)$ be the projective algebra of all subsets of ${ }^{2} U_{j}$ with atom $\{\langle a, a\rangle\}$. The Boolean algebra with the following atoms is easily seen to be a projective subalgebra of $\mathfrak{A}\left(U_{j}\right)$ :

$$
\begin{aligned}
& f_{0 j}=\{\langle a, a\rangle\}, \quad f_{1 j}=\left\{\langle u, u\rangle: u \in U_{j}, u \neq a\right\}, \\
& f_{2 j}=\left\{\langle a, v\rangle: v \in U_{j}, v \neq a\right\}, \quad f_{3 j}=\left\{\langle u, a\rangle: u \in U_{j}, u \neq a\right\},
\end{aligned}
$$

and

$$
f_{4 j}=\left\{\langle u, v\rangle: u, v \in U_{j} \sim\{a\}, u \neq v\right\} .
$$

Denote this subalgebra by $\mathfrak{B}_{j}$. Then obviously there is a projective algebra $\mathfrak{M}$ and isomorphisms $g_{j}$ onto $\mathfrak{B}_{j}$ such that $g_{1} g_{0}{ }^{-1} D_{0}=D_{1}$. Thus (A) holds and Theorem 1 applies.

COROLLARY 5. The embedding property fails for the following classes of algebras where $1<\alpha<\omega$ : $R C A^{\prime}$ s and $C A_{\alpha}{ }^{\prime}$ s of characteristic 0 , (representable) polyadic equality algebras of dimension $\alpha$ and characteristic 0 , and $R R A^{\prime} s$ and $R A^{\prime}$ s of class 3 .

For $1<\alpha<\omega$ the classes of $C A_{\alpha}{ }^{\prime}$ s and polyadic equality algebras of dimension $\alpha$ with a fixed nonzero characteristic and the classes of $R A^{\prime} s$ with class $\neq 3$ are known to have the embedding property. In fact, these classes have the amalgamation property (cf., Comer [1]). By essentially the same argument given on p. 226 of Halmos [5] the embedding property can be shown to hold for the class of projective algebras, $R C y_{\alpha}$, and the class of representable polyadic algebras of dimension $\alpha$ where $1<\alpha<\omega$. Whether or not this property holds for the class $C y_{\alpha}$ where $1<\alpha<\omega$ and for the class of all polyadic algebras of dimension $\alpha$ where $2<\alpha<\omega$ still appears to be open.

By examining the proof of Lemma 3 we see that if we restrict ourselves to the category of all complete $C A_{\alpha}{ }^{\prime}$ s with complete homomorphisms, then the amalgamation and embedding properties fail for all $\alpha>1$. Similar results hold for the other classes of algebras listed in Corollaries 4 and 5 if we modify the category.

For the sake of completeness we will also consider the amalgamation property for the classes $C y_{\alpha}$ and $C A_{\alpha}$ for $\alpha \leqq 1$ (and hence also for the classes of all polyadic and polyadic equality algebras of dimension $\alpha \leqq 1$ ).

THEOREM 6. For $\alpha \leqq 1$ the amalgamation property holds for the classes $C A_{\alpha}$ and $C y_{\alpha}$. 
Proof. For $\alpha=0$ the algebras to be considered are just Boolean algebras so the conclusion follows from Dwinger and Yaqub [3]. For $\alpha=1$ notice first that we can clearly amalgamate the simple algebras of each of the classes. The amalgamation property for the classes now follows by the same argument as used in Theorem 2.7 of Daigneault [2] for the class of all locally-finite polyadic algebras of infinite dimension.

3. We conclude the paper by establishing in Theorem 8 a sufficient (and obviously necessary) condition for two given algebras of a class $K$ to be embeddable in some algebra of $K$. Conditions of a different nature may be found in $\operatorname{Los}[10],[11]$. As a corollary of Theorem 8 we show that the hypothesis $\left|U_{0}\right|<\omega$ is necessary for the conclusion of Lemma 3 and Theorem 2. More precisely:

Corollary 7. If $\left|U_{0}\right|,\left|U_{1}\right| \geqq \omega$ and $1<\alpha<\omega$, then we can embed the full set $C y_{\alpha}{ }^{\prime} s \mathfrak{A}_{c}\left(\alpha, U_{0}\right)$ and $\mathfrak{A}_{c}\left(\alpha, U_{1}\right)$ in a $C y_{\alpha}$ such that the main diagonals of $\mathfrak{A}\left(\alpha, U_{0}\right)$ and $\mathfrak{N}\left(\alpha, U_{1}\right)$ are mapped to the same element.

We need the following definition. For the general notion of reduct consult Tarski [14]; for the notion restricted to cylindric algebras as well as the notion of a neat embedding consult Henkin and Tarski [6]. Suppose the similarity type $\mu$ is an expansion of the similarity type $\tau$, i.e., dom $\tau \leqq \operatorname{dom} \mu$ and $\mu \mid \operatorname{dom} \tau=\tau$. A class $L$ of algebras with similarity type $\mu$ is called a $\mu$-extension of a class $K$ of algebras of type $\tau$ if every $\tau$-reduct of an algebra in $L$ belongs to $K$. We donote the $\tau$-reduct of $\mathscr{A} \in L$ by $\mathrm{Rd}_{\tau} \mathscr{A}$.

THEOREM 8. Suppose $K$ is a class of similar algebras of type $\tau$ and $\mathfrak{A}, \mathfrak{B} \in K . \quad A$ sufficient (and obviously necessary) condition for there to exist $\mathfrak{E} \in K$ such that $\mathfrak{A}$ and $\mathfrak{B}$ are embeddable in $\mathfrak{F}$ is that there exist a $\mu$-extension $K^{\prime}$ of $K$ for some expansion $\mu$ of $\tau$ and algebras $\mathfrak{H}^{\prime}$ and $\mathfrak{B}^{\prime}$ in $K^{\prime}$ such that

(i ) $\mathfrak{A} \nrightarrow \mathrm{Rd}_{\tau} \mathfrak{X}^{\prime}$ and $\mathfrak{B} \nrightarrow \mathrm{Rd}_{\tau} \mathfrak{B}^{\prime}$;

(ii) There exist $\mathbb{(}^{\prime} \in K^{\prime}$ such that $\mathfrak{X}^{\prime}, \mathfrak{B}^{\prime}$ are embeddable in $\mathfrak{\mho}^{\prime}$.

Proof. Suppose we are given $\mu, K^{\prime}, \mathfrak{X ^ { \prime }}, \mathfrak{B}^{\prime}$ as in the statement. By (ii) there is a $\mathbb{C}^{\prime} \in K^{\prime}$ such that $\mathfrak{Y}^{\prime} \nrightarrow \mathbb{C}^{\prime}$ and $\mathfrak{B}^{\prime} \nrightarrow \mathfrak{C}^{\prime}$. By (i) and properties of reducts $\mathfrak{A}$ and $\mathfrak{B}$ are embeddable in $\mathrm{Rd}_{\tau} \mathfrak{S}^{\prime}$ which belongs to $K$ since $K^{\prime}$ is a $\mu$-extension of $K$.

Proof of Corollary 7. First observe that it suffices to embed the $C A_{\alpha}{ }^{\prime} \mathrm{s} \mathfrak{A}\left(\alpha, U_{0}\right)$ and $\mathfrak{A}\left(\alpha, U_{1}\right)$ in some $C A_{\alpha}$. To do this we apply 
Theorem 8 with $K=C A_{\alpha}$ and $K^{\prime}$ the class of locally-finite $C A_{\omega}{ }^{\prime}$ s. It is clear that $K^{\prime}$ is a $\mu$-extension of $K$ where $\mu$ is the similarity type of $K^{\prime} . \mathfrak{N}\left(\alpha, U_{0}\right)$ and $\mathfrak{U}\left(\alpha, U_{1}\right)$ can be neatly embedded (cf., Theorem 1.2 of Monk [13]) in locally-finite subalgebras $\mathfrak{U}^{\prime}$ of $\mathfrak{A}\left(w, U_{0}\right)$ and $\mathfrak{B}^{\prime}$ of $\mathfrak{A}\left(\omega, U_{1}\right)$, respectively. Hence we have $\mathfrak{U}^{\prime}$ and $\mathfrak{B}^{\prime}$ for which 8 (i) holds. Since $\left|U_{0}\right|,\left|U_{1}\right| \geqq \omega$, $\mathfrak{X}^{\prime}$ and $\mathfrak{B}^{\prime}$ have isomorphic minimal subalgebras; thus by the amalgamation property for locally-finite $C A_{\omega}{ }^{\prime}$ s condition 8 (ii) holds. The corollary now follows.

Another obvious consequence of Theorem 8 is that if $K$ is a class of similar algebras of type $\mu$ which has the embedding property, then the class $\operatorname{Rd}_{\tau} K=\left\{\operatorname{Rd}_{\tau} \mathfrak{I}: \mathfrak{A} \in K\right\}$ also has the embedding property, where $\tau$ is any restriction of $\mu$.

I wish to thank the referee for his suggested improvements and his contribution to Corollary 4.

\section{REFERENCES}

1. S. Comer, Galois theory and the amalgamation property in finite dimensional cylindric algebras, Preliminary report, Amer. Math. Soc. Notices 15 (1968), 103.

2. A. Daigneault, On automorphisms of polyadic algebras, Amer. Math. Soc. Trans. 112 (1964), 84-130.

3. P. Dwinger and F. M. Yaqub, Generalized free products of Boolean algebras with an amalgamated subalgebra, Indag. Math. 25 (1963), 225-231.

4. C. J. Everett and S. M. Ulam, Projective algebras I, Amer. J. Math. 68 (1946), $77-88$.

5. P. R. Halmos, Algebraic logic, Chelsea, New York, 1962.

6. L. Henkin, and A. Tarski, Cylindric algebras, Lettice Theory, Proc. Sympos. Pure. Math., Vol 2, Amer. Math. Soc., Providence R. I., 1961.

7. J. S. Johnson, Amalgamation of polyadic algebras, Preliminary report, Amer. Math. Soc. Notices, 14 (1967) 361.

8. B. Jónsson, Extensions of relational structures, The Theory of Models, Proc. 1963 Int. Sympos. at Berkeley, North-Holland Pub. Co., Amsterdam, 1965.

9. B. Jónsson, and A. Tarski, Boolean algebras with operators $I$ and II, Amer. J. Math. 73 (1951), 891-939; 74 (1952), 127-167.

10. J. Los, Quelques Remarques, Theorems et Problemes sur les Classes Definissables d'Algebres, Mathematical Interpretation of Formal Systems, North-Holland Pub. Co., Amsterdam, 1955.

11. - Common extension in eguational classes, Logic Methodology and Philosophy of Science, Proceedings of the 1960 International Congress, Stanford University Press, Stanford, 1962,

12. R. McKenzie, The representation of relation algebras, Doctoral dissertation, University of Colorado, 1966.

13. D. Monk, Model-thoretic methods and results in the theory of cylindric algebras, The Theory of Models, Proc. 1963 Int. Sympos, at Berkeley, North-Holland Pub. Co., Amsterdam, 1965.

14. A. Tarski, Contributions to the theory of models, I., Kon. Ned. Akad. van Wet., 
Proc., Ser. A 57 (1954), 572-581; (also, Indag. Math. 16).

Received February 22, 1968. This paper is adapted from $\& 2$ of the author's doctoral dissertation. The research was supported by a predoctoral NSF Traineeship and it was written under NSF Grant GP 7252.

VANDERBILT UNIVERSITY

NashVille, TENNESSEe 


\section{PACIFIC JOURNAL OF MATHEMATICS}

\section{EDITORS}

H. ROYDEN

Stanford University

Stanford, California

\section{R. $R$ PHELPS}

University of Washington

Seattle, Washington 98105

\section{J. DugunduI}

Department of Mathematics

University of Southern California

Los Angeles, California 90007

RICHARD ARENS

University of California

Los Angeles, California 90024

\section{ASSOCIATE EDITORS}

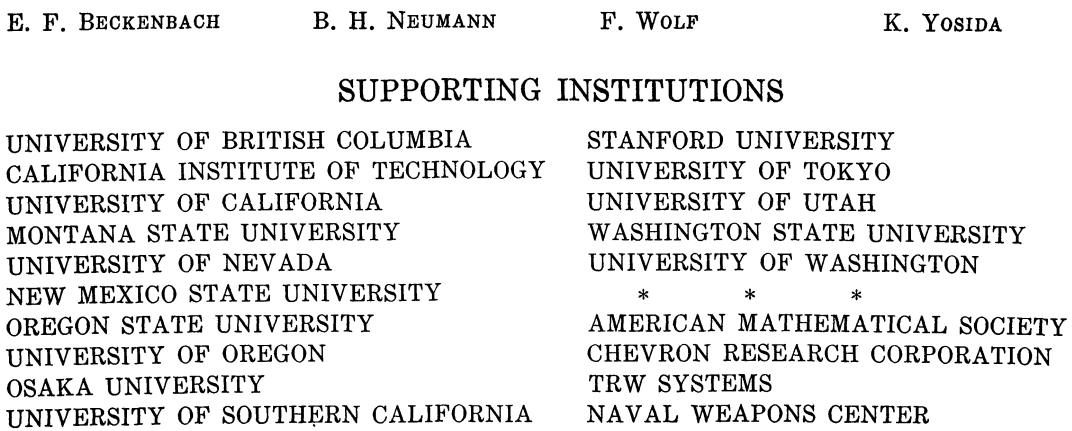

The Supporting Institutions listed above contribute to the cost of publication of this Journal, but they are not owners or publishers and have no responsibility for its content or policies.

Mathematical papers intended for publication in the Pacific Journal of Mathematics should be in typed form or offset-reproduced, double spaced with large margins. Underline Greek letters in red, German in green, and script in blue. The first paragraph or two must be capable of being used separately as a synopsis of the entire paper. It should not contain references to the bibliography. Manuscripts, in duplicate if possible, may be sent to any one of the four editors. Please classify according to the scheme of Math. Rev. 36, 1539-1546. All other communications to the editors should be addressed to the managing editor, Richard Arens, University of California, Los Angeles, California, 90024.

50 reprints are provided free for each article; additional copies may be obtained at cost in multiples of 50 .

The Pacific Journal of Mathematics is published monthly. Effective with Volume 16 the price per volume (3 numbers) is $\$ 8.00$; single issues, $\$ 3.00$. Special price for current issues to individual faculty members of supporting institutions and to individual members of the American Mathematical Society: $\$ 4.00$ per volume; single issues $\$ 1.50$. Back numbers are available.

Subscriptions, orders for back numbers, and changes of address should be sent to Pacific Journal of Mathematics, 103 Highland Boulevard, Berkeley, California, 94708.

PUBLISHED BY PACIFIC JOURNAL OF MATHEMATICS, A NON-PROFIT CORPORATION

Printed at Kokusai Bunken Insatsusha (International Academic Printing Co., Ltd.), 7-17, Fujimi 2-chome, Chiyoda-ku, Tokyo, Japan. 


\section{Pacific Journal of Mathematics}

Vol. 28, No. $2 \quad$ April, 1969

Richard Arens and Donald George Babbitt, The geometry of relativistic

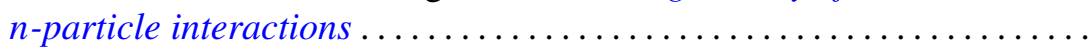

Kirby Alan Baker, Hypotopological spaces and their embeddings in lattices

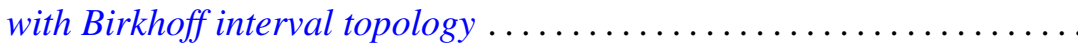

J. Lennart (John) Berggren, Finite groups in which every element is conjugate to its inverse ........................... 289

Beverly L. Brechner, Homeomorphism groups of dendrons . . . . . . . . . . . 295

Robert Ray Colby and Edgar Andrews Rutter, QF - 3 rings with zero singular ideal ................................. 303

Stephen Daniel Comer, Classes without the amalgamation property....... 309

Stephen D. Fisher, Bounded approximation by rational functions ......... 319

Robert Gaines, Continuous dependence for two-point boundary value problems..................................... 327

Bernard Russel Gelbaum, Banach algebra bundles ............... 337

Moses Glasner and Richard Emanuel Katz, Function-theoretic degeneracy criteria for Riemannian manifolds ...................... 351

Fletcher Gross, Fixed-point-free operator groups of order $8 \ldots \ldots \ldots \ldots 357$

Sav Roman Harasymiv, On approximation by dilations of distributions . . . . 363

Cheong Seng Hoo, Nilpotency class of a map and Stasheff's criterion ... . . 375

Richard Emanuel Katz, A note on extremal length and modutus.......... 381

H. L. Krall and I. M. Sheffer, Difference equations for some orthogonal polynomials ................................

Yu-Lee Lee, On the construction of lower radical properties ........... 393

Robert Phillips, Liouville's theorem........................... 397

Yum-Tong Siu, Analytic sheaf cohomology groups of dimension $n$ of

n-dimensional noncompact complex manifolds ..... . .

Michael Samuel Skaff, Vector valued Orlicz spaces. II...

James DeWitt Stein, Homomorphisms of $B^{*}$-algebras .... . .

Mark Lawrence Teply, Torsionfree injective modules .... . . .

Richard R. Tucker, The $\delta^{2}$-process and related topics. II .

David William Walkup and Roger Jean-Baptiste Robert Wets, Lifting

projections of convex polyhedra...

Thomas Paul Whaley, Large sublattices of a lattice. 